Catherine LECLERCQ, Wenceslas LIZÉ, Hélène STEVENS, dirs, Bourdieu et les sciences sociales. Réception et usages

Paris, Éd. La Dispute, coll. Mouvements de société, 2015, 344 pages

\title{
Emmanuel Brandl
}

\section{(2) OpenEdition}

\section{Journals}

Édition électronique

URL : https://journals.openedition.org/questionsdecommunication/11387

DOI : 10.4000/questionsdecommunication. 11387

ISSN : 2259-8901

Éditeur

Presses universitaires de Lorraine

\section{Édition imprimée}

Date de publication : 1 septembre 2017

Pagination : 564-567

ISBN : 9782814303256

ISSN : 1633-5961

\section{Référence électronique}

Emmanuel Brandl, «Catherine Leclerca, Wenceslas lizé, Hélène stevens, dirs, Bourdieu et les sciences sociales. Réception et usages », Questions de communication [En ligne], 31 | 2017, mis en ligne le 01 septembre 2017, consulté le 26 octobre 2021. URL : http://journals.openedition.org/ questionsdecommunication/11387 ; DOI : https://doi.org/10.4000/questionsdecommunication 11387 
psychanalyse freudienne, de Sigmund Freud à Jacques Lacan (pp. 99-131). La seconde, sobrement mais ambitieusement intitulée tout de même « Le Langage poétique » (pp. | 33-25 I), s'attache à reconstituer dans les notes et les ouvrages d'Émile Benveniste non l'objet que serait le langage poétique objectivé, mais la démarche subjectivisante qui tend à l'instancier. Le problème et le poème (pp. |33-148) revient sur la résonance paronomastique de ces deux termes et, de cette résonance, fait en quelque sorte l'étymon spirituel de la démarche du linguiste. Est ensuite abordée la question, de la relation du Poème au réalisme linguistique (pp. I 49$\mid 91$ ). Sous « Un cosmos nouveau et spécifique » (pp. 193-200), Chloé Laplantine aborde la question qui a tant ému le microcosme des linguistes : la linguistique est-elle ou n'est-elle pas une science pilote des autres sciences humaines; question à laquelle Émile Benveniste répond par la négative, préférant avancer sa conception d'une culturologie dans laquelle la société est proprement la dynamique de sa production par le langage (p. 194). Avec « La Poésie dans le langage ordinaire » (pp. 201226), est posée la distinction fondamentale qui fait du langage poétique un être entièrement différent du langage ordinaire par l'obligation qu'il a d'être subjectivant en créant une « sémiologie nouvelle » (p. 205). C'est dire que les limites et le niveau de l'analyse linguistique traditionnelle ne sont plus ici de mise. « Le syntagme au-delà de ses limites » (pp. 227-236) insiste à juste titre sur cet aspect et sur la recherche qu'a développée Émile Benveniste pour essayer de trouver une notion qui rende compte de cette expansion à travers divers néologismes essayés : sympathème, symphrorie, symphronie. Dans « Un langage iconique » (pp. 237-250), Chloé Laplantine rappelle la différence fondamentale opposant Émile Benveniste à Charles S. Peirce sur la question de l'icône. Ce dernier fait de l'icône un objet statique dont la qualité essentielle en tant que signe est le réalisme puisque le signe renvoie à un réel non interrogé (p. 248) ; tandis qu'Émile Benveniste oppose le signe linguistique commun à tous et conceptuel à l'icône unique et émotionnel(le) tel que le poème le/la fait advenir dans son intenté (p. 246). Une brève conclusion (pp. 249-25I) resserre les fils de cet important travail en montrant qu'Émile Benveniste emporte avec sa réflexion une théorie du langage entièrement différente de celle qui prévaut chez la plupart des linguistes étudiant le langage comme un produit, ergon, plutôt que comme une dynamique, energeia... Ce faisant, et sans avoir besoin de remonter jusqu'à Wilhelm von Humboldt, Émile Benveniste est à même de montrer que « bien avant de servir à communiquer, le langage sert à vivre » (Problèmes de Linguistique générale, Paris, Gallimard, 1974 : 217, cité p. 250). Et c'est là, probablement, ce que le livre de Chloé Laplantine met le mieux en évidence.
On relèvera d'ailleurs à ce sujet, de manière sympathique mais inattendue dans le cadre d'un travail de recherche, les multiples incidentes subjectives dont elle parsème son discours, qui, en tant que telles, redoublent la forte prise en charge énonciative de son propos : selon moi, d'après moi, à mon sens, il me semble, je pense... II n'y a aucun intérêt à fonder sur un structuralisme désuet, et fondamentalement extérieur à toute historicité du sujet - et pas seulement obnubilé par l'éviction de l'histoire - l'étude de cet objet vivant qu'est le langage, notamment lorsque celui-ci se développe dans sa dimension poétique faisant apparaitre le lien infrangible unissant le medium et ses médiateurs dans l'acte de création de la signifiance que requiert le poème. On comprend mieux par-là que le langage ordinaire n'est tout au plus qu'une réduction du Langage dans toute la puissance que celui-ci concentre, et qui se diffracte en discours sous les couleurs de l'éthique, du politique, du sociologique, de l'anthropologique. Les deux annexes qui accompagnent cet ouvrage : « Documents : Le fonds Émile Benveniste » (pp. 255-272), puis « Références bibliographiques » (pp. 273-283) ajoutent à l'intérêt indéniable de ce travail que deux indices (noms de personnes et notions) achèvent de constituer en très appréciable outil de travail puisque c'est le vœu de Chloé Laplantine que de voir sa recherche prolongée par d'autres soucieux de « poursuivre en allant voir plus loin de quelle poétique Benveniste nous rend capable » (p. 252). Elle même prolongeant, comme Jacques Fontanille a pu le faire pour Algirdas Julien Greimas, les travaux de Gérard Dessons et d'Henri Meschonnic dans leur coruscante critique de tout ce qui dénie au langage sa dimension anthropologique.

Jacques-Philippe Saint-Gerand CeReS, université de Limoges, F-87000 jacques-philippe.saint-gerand@unilim.fr

Catherine LeCLERCQ, Wenceslas LIzé, Hélène Stevens, dirs, Bourdieu et les sciences sociales. Réception et usages Paris, Éd. La Dispute, coll. Mouvements de société, 2015, 344 pages

Bourdieu et les sciences sociales. Réception et usages est la publication des actes d'une journée d'étude qui s'inscrit dans une entreprise de constitution d'une histoire sociale des sciences sociales (avec par exemple l'ouvrage de Johan Heilbron, Rémi Lenoir et Gisèle Sapiro, éds., Pour une histoire des sciences sociales. Hommage à Pierre Bourdieu, Paris, Fayard, 2004). L'ouvrage se compose de trois parties qui questionnent les usages de Pierre Bourdieu dans des disciplines différentes en partant du plus « proche » 
pour aller vers le plus « lointain ». De la sorte, la première partie est consacrée aux appropriations de Pierre Bourdieu en sociologie, quand la deuxième porte un regard sur des disciplines dans lesquelles ce dernier est installé et discuté (anthropologie, science politique, études littéraires), tandis que la troisième s'intéresse à des disciplines où il fait encore l'objet d'une réception limitée (sciences de l'information et de la communication, économie, droit, histoire médiévale, histoire moderne en Allemagne). L'ouvrage se présente comme une « étude de la réception différenciée » (p. 9) de Pierre Bourdieu, tout en souhaitant s'émanciper de l'esprit de discipline pour penser l'unité des sciences sociales. C'est en ce sens que l'introduction de l'ouvrage est prolongée d'un texte inédit du sociologue intitulé « Sur la possibilité d'un champ international de la sociologie » (pp. 33-49).

Globalement, si la dimension réflexive qu'enferme chacune des contributions qui alimentent l'ouvrage donne une tonalité et un intérêt particulièrement stimulant à cette publication, il reste que la particularité de ce livre réside dans les deux longues introduction (pp. 7-32) et conclusion (pp. 305-332) rédigées par Catherine Leclercq, Wenceslas Lizé et Hélène Stevens. En effet, il est courant que l'introduction et/ou la conclusion d'un ouvrage publiant les actes d'un colloque ou d'une journée d'étude restitue la problématique qui a réuni les auteurs concernés/présents et, parfois même, une brève synthèse de chacune des contributions. Ici, l'introduction comme la conclusion font l'objet d'un véritable travail d'analyse des différentes contributions prises comme autant de documents permettant d'étudier les usages récurrents et spécifiques de Pierre Bourdieu, à produire aussi une synthèse des manières dont tel ou tel concept ou précepte a été réapproprié, à essayer de mieux cerner aussi les freins et les conditions favorables de réception de cet auteur dans les disciplines citées. Le fil conducteur de leur travail a été d'interroger les conditions de possibilité d'une appropriation des outils théoriques et méthodologiques de Pierre Bourdieu au prisme de sa trajectoire sociale et intellectuelle mais aussi des transformations du champ scientifique depuis les années 50.

Ainsi apparaît-il que l'état du champ scientifique dans les années 60 (lui aussi marqué par l'épisode « mai 68 ») est favorable à une « humeur anti-institutionnelle » dans laquelle Pierre Bourdieu se retrouve pleinement: le champ scientifique définit un espace des possibles, un espace de positions scientifiques composé de propositions théoriques et méthodologiques qui sont dominantes et qu'il faut à ce moment-là dépasser pour transformer la structure du champ (donc la structure des positions dominantes) en transformant « les critères de la production scientifique et plus généralement les règles de fonctionnement du champ académique »- p. 312 (Claude Lévi-Strauss avec le structuralisme, Jean-Paul Sartre avec l'existentialisme, Michel Foucault avec la notion d'épistémé, etc. - travail d'analyse entrepris par Louis Pinto dans Pierre Bourdieu et la théorie du monde social, Paris, A. Michel, 1999). De plus, sa trajectoire sociale, d'une origine sociale basse à une reconnaissance intellectuelle internationale à travers un parcours scolaire d'excellence, le prédispose à une certaine ambition scientifique pour la sociologie (il piaffait « d'en découdre "théoriquement" avec ses aînés », p. 75), mais aussi à adopter un point de vue sur le champ scientifique qui l'amène à avoir une certaine « lucidité », comme manière de voir certaines frontières (disciplinaires, méthodologiques...) et à les penser comme arbitraires. Pierre Bourdieu incarne cet « habitus clivé » (Gérard Mauger, « Le style de pensée de Pierre Bourdieu », pp. 53-7I) qui prédispose les individus qui en sont porteurs à se positionner aux frontières des champs. En l'occurrence, Pierre Bourdieu ne cessera pas de rompre avec les cloisonnements scientifiques (disciplinaires et méthodologiques), avec les formes d'académisme (jusqu'à l'insistance qu'il met dans un ouvrage comme Méditations pascaliennes à critiquer les présupposés scolastiques de l'intellectuel - Pierre Bourdieu, Médiations pascaliennes, Paris, Éd. Le Seuil, 1997), et à chercher à allier les contraires: subjectivisme et objectivisme, analyse externe et analyse interne, ethnologie et sociologie, aller vers la sociologie - et avant l'ethnologie par son séjour en Algérie - alors qu'il est agrégé de philosophie, etc. Cela le conduira à forger des outils conceptuels « médiateurs », qui permettent de penser par exemple l'articulation des structures sociales avec l'individu luimême, à travers le concept d'habitus.

Les auteurs rappellent ensuite que les modalités de réception de Pierre Bourdieu ne s'arrêtent pas à cette « humeur » dans laquelle des chercheurs de disciplines différentes peuvent se reconnaitre (reconnaissance quills doivent eux-mêmes à leur trajectoire sociale). Elles doivent aussi beaucoup au processus d'accumulation d'un « capital symbolique » de Pierre Bourdieu, accumulé à travers des «s stratégies 》 (au sens de Pierre Bourdieu) scientifiques donc, mais aussi éditoriales (direction d'une collection aux éditions de Minuit, puis Le Seuil, création de la revue Actes de la recherche en sciences sociales...) et institutionnelles (Raymond Aron lui confie le direction du Centre de sociologie européenne, il obtient le premier poste de sociologie au Collège de France), des différentes formes de consécration (au niveau national, il obtiendra la première médaille d'or du 
CNRS de l'histoire de la sociologie), suivis d'engagements publics (dans les années 90). C'est alors que Catherine Leclercq, Wenceslas Lizé et Hélène Stevens opèrent un rapprochement systématique des périodes de réception mentionnées par les contributeurs de l'ouvrage en lien avec cette trajectoire. Ils peuvent ainsi montrer que c'est dans les années 90 que la réception de Pierre Bourdieu est la plus forte : son œuvre est reconnue de plein droit (cette synchronie apparait dans les contributions de Bastien Bosa, « Usages des travaux de Bourdieu en anthropologie », pp. | |7- | 35 ; Paul Dirkx, « Bourdieu et les études littéraires », pp. |6|-|8| ; Bernard Pudal et Boris Gobille, « Deux appropriations de Bourdieu en science politique », pp. 137-160; Frédéric Lebaron, «Bourdieu et l'économie : quel dialogue? », pp. 205 224 et Bruno Ambroise, « Bourdieu en philosophe », pp. 283-303).

Les coordinateurs de l'ouvrage proposent aussi une typologie des usages scientifiques de Pierre Bourdieu qui dépasse les appartenances disciplinaires. Quatre types d'usages sont répertoriés:

I/ L'usage référentiel : il consiste moins à user des concepts de Pierre Bourdieu qu'à user des réflexions critiques ou méthodologiques qu'il produit pour les mettre en œuvre dans son propre domaine de recherche (Malha Naab, « Bourdieu, le droit et les juristes », pp. 225-245).

2/ L'usage instrumental :il consiste à considérer l'outillage conceptuel et méthodologique de Pierre Bourdieu comme une boite à outil. Ce type d'usage est, aux yeux même de Pierre Bourdieu, problématique, puisqu'il autonomise chaque concept des autres (《habitus 》, 《 champ 》, 《 capital », 《 illusio 》, « dispositions ») alors qu'il s'agit, pour leur auteur, de concepts interdépendants. Pourtant, de nombreux chercheurs - tel Boris Klein dans le livre ( « Pour un usage de Bourdieu en histoire moderne », pp. 265-28I) - appellent de leurs vœux un usage « décomplexé » de la pensée de Pierre Bourdieu.

3/ L'usage structurant : il consiste à l'inverse à user pleinement de tout ou partie des concepts et méthodologies de Pierre Bourdieu, comme le concept de « champ littéraire » pour les « études littéraires 》 (« Bourdieu et les études littéraires », pp. |6|-|8|), ou d'habitus en anthropologie ( « Usages des travaux de Bourdieu en anthropologie », pp. |17-135). Ce type d'usage participe pour certains auteurs d'une redéfinition de leurs objets de recherche et de leur légitimité dans le champ scientifique.
4/ L'usage innovant : il consiste à travailler les concepts de Pierre Bourdieu au regard de nouvelles questions, de nouveaux terrains d'enquête ou de contextes historiques inédits (Jean-Philippe Genet, « Usages du travail de Bourdieu en histoire médiévale », pp. 247 263 , mais on pourra aussi se reporter aux travaux de Bernard Lahire sur la pluralité des dispositions individuelles ou encore à ceux de l'économiste Frédéric Lordon autour du concept de « conatus »).

Les auteurs relèveront très justement que la notion de « réflexivité »(Stéphane Beaud et Michel Pialoux, «Partir à Sochaux avec "Bourdieu en tête" », pp. 73-94 ; Bertrand Geay, « Faut-il casser les amphithéâtres? », pp. 95- | | 3) fait, quant à elle, l'objet d'un usage transversal. En effet, Pierre Bourdieu a installé progressivement dans les sciences sociales, dès les années 70, une « humeur réflexive » (p. 328) à plusieurs niveaux, dont trois sont relevés:

I/ Au niveau des classements sociaux et des catégories d'analyse : Pierre Bourdieu propose d'interroger la genèse des catégories d'analyse, comme des modes de classification et d'institutionnalisation, en revenant sur les luttes de classement pour dénaturaliser ces catégories.

2/ Au niveau des méthodologies d'enquête : il s'agit d'expliciter les conditions d'enquête, d'analyser les sources, d'analyser les conditions d'utilisation de techniques variées (statistique, entretien, observation. . .).

3/ Au niveau, enfin, du chercheur lui-même : c'est l'objectivation du sujet objectivant qui doit être réalisée en analysant l'état du champ, la trajectoire et la position occupée par le chercheur dans le champ, mais aussi en critiquant l'impensé scolastique de chaque discipline, ce qui revient à en critiquer la doxa, ici celle de l'idéologie d'une pensée pure (Jean-Paul Sartre définissant ainsi un individu « sans attaches, ni racines », libre de sa propre vérité).

Cette critique réflexive a souvent été perçue comme une dépréciation des autres disciplines des sciences humaines et sociales par Pierre Bourdieu (Stéphane Olivesi, « Une rencontre manquée : Bourdieu et les SIC [sciences de l'information et de la communication] », pp. 185-204). Elle a été l'un des freins, avec les obstacles pédagogiques (liés à la complexité sémantique et syntaxique des écrits de Pierre Bourdieu), à la réception de son œuvre. À ces obstacles « pédagogiques 》 et « scolastiques 》, Catherine Leclercq, Wenceslas Lizé et Hélène Stevens en ajoutent un troisième, l'obstacle « politique ». II s'agit ni plus ni moins d'une conséquence de l'objet même des analyses de Pierre Bourdieu, soit les mécanismes de la domination. Mais d'autres obstacles, méthodologiques, interviennent: 
la difficulté à penser des époques révolues avec des catégories d'analyse construites pour appréhender des périodes plus récentes, comme c'est le cas avec le concept de « champ ». Pourtant, un historien médiéviste comme Jean-Philippe Genet (« Usages du travail de Bourdieu en histoire médiévale », pp. 247-263) s'approprie le concept, tout en le déclinant de façon innovante en différentes catégories : « champ englobant 》, champ 《 structuré par l'ajustement à des "institutions" » et « champs composites 》 - c'est aussi une des originalités de l'ouvrage que de restituer certains de ces usages méconnus de la pensée de Pierre Bourdieu, comme en droit et en histoire médiévale. Enfin, en dehors des effets de réforme (réformes politiques récentes qui fragilisent l'autonomie des enseignants-chercheurs - peu rentables - et bousculent la table des valeurs professionnelles comme des critères de légitimité), l'institutionnalisation des disciplines est aussi un frein, car à la source même de la cristallisation des frontières disciplinaires (Stéphane Olivesi, « Une rencontre manquée : Bourdieu et les SIC », pp. 185-204). Malgré cela, on voit aujourd'hui se constituer « un espace à l'intersection de plusieurs disciplines au sein duquel les chercheurs ont en commun les emprunts à la sociologie et en particulier à celle de Bourdieu » (p. 33I), notamment grâce à Pierre Bourdieu lui-même qui a souvent réalisé « le travail de traduction du dépassement de ces oppositions selon les catégories de leur champ disciplinaire » (p. 3/3).

II convient donc de saluer cette publication, d'abord parce que l'ouvrage va au-delà du fait de réunir des auteurs pour parler de Pierre Bourdieu : elle apporte un éclairage sur «Bourdieu » qui relève de l'analyse sociologique. Ensuite, en travaillant au dévoilement des conditions sociales et scientifiques de possibilité de production d'un espace interdisciplinaire de la recherche en sciences sociales, elle produit un effet stimulant et invite plus que jamais à suivre une telle piste de réflexion pour le meilleur de la recherche, en général (à l'image de Bernard Lahire et Claude Rosental, dirs, La Cognition au prisme des sciences sociales, Paris, Éd. des Archives contemporaines, 2008).

Emmanuel Brandl

Centre Max Weber, Enssib, F-69100 emmanuel.brand@enssib.fr

\section{Pierre MÆGLIN, dir., Industrialiser l'éducation. Anthologie commentée (19/3-2012)}

Saint-Denis, Presses universitaires de Vincennes, coll. Médias, 2016, 388 pages

II y a exactement 30 ans que Pierre Mœglin signait dans le Bulletin de l'DATE sa première publication sur la thématique de l'industrialisation de l'éducation
(« La notion de besoins éducatifs : remarques sur l'introduction des nouveaux médias dans l'éducation 》, Bulletin de l'IDATE, 19, 1986, pp.89-99 »). Autant dire que ce livre, qui se présente très, ou trop modestement, comme une anthologie, constitue, de la part de Pierre Mœglin, une somme et une synthèse impressionnantes d'années de recherche, ponctuées par une quinzaine de publications marquantes sur ce sujet méconnu.

Méconnu, en France plus qu'outre-Atlantique, sans doute du fait de la prégnance, en France, d'une conception d'abord culturelle de l'éducation, fortement opposée à l'idée même d'une industrialisation de l'éducation, perçue ici comme un oxymore. Pourtant il y a près de cent ans que Joseph Wilbois, dans La Nouvelle Éducation française (Paris, Payot, 1922), mettait l'accent sur la rationalisation industrielle des méthodes d'éducation. Et il est bien rare aujourd'hui de trouver un universitaire qui ne souligne la croissance du fait gestionnaire à l'université, ou la multiplicité des procédures de contrôle et d'évaluation des contrats de tous types, sans forcément mettre cela en relation avec une rationalisation de type industriel.

Cette méconnaissance est d'autant plus étonnante que deux autres logiques, que Jean-Louis Derouet nomme « logique civique » et « logique domestique » (pp. 237-246) sont partout rappelées avec force : pour exemple, la revue grand public Le Point Références, dans sa livraison d'oct-nov. 2016, L'éducation idéale. Les textes fondamentaux, consacre sa partie centrale au « siècle des innovations », avec John Dewey, Rudolf Steiner, Édouard Claparède, Jean Piaget, Célestin Freinet, Maria Montessori ou Alexander S. Neill, sans un mot pour les pionniers de ce qu'il faut bien nommer industrialisation. En France, il est vrai, la « logique civique » a une longue histoire, depuis le Rapport et projet de décret sur l'organisation générale de l'instruction publique de Condorcet en 1792, dont nous citons ici le principe : «Établir entre les citoyens une égalité de fait, et rendre réelle l'égalité politique [...] tel doit être le premier but d'une instruction nationale »; depuis Jules Ferry, qui s'en inspire directement, jusqu'au « collège unique » contemporain, cette logique (l'école de la République) n'a cessé d'être réactivée, en concurrence avec la logique « domestique » qu'alimente l'idée de « communauté scolaire » ou de l'école comme « lieu de vie ». Le phénoménal succès des Libres enfants de Summerhill (Alexander S. Neill, trad. de l'anglais par M. Laguilhomie, Paris, F. Maspero, 1970), en 1971, en dit long sur l'occultation durable de la réflexion sur le triple pilier technologique, rationnel, et idéologique d'une « industrialisation éducative » comme autre modèle de l'éducation. 